\title{
A Comprehensive Analysis of the circRNA-miRNA-mRNA Network in Osteocyte-Like Cell Associated with Mycobacterium Leprae Infection
}

\section{Zheng-Rong Gao}

Second Xiangya Hospital of Central South University

\section{Qiong Liu}

Second Xiangya Hospital of Central South University

\section{Jie Zhao}

Second Xiangya Hospital of Central South University

\section{Ya-Qiong Zhao}

Second Xiangya Hospital of Central South University

Li Tan

Second Xiangya Hospital of Central South University

Shao-Hui Zhang

Second Xiangya Hospital of Central South University

\section{Ying-Hui Zhou}

Second Xiangya Hospital of Central South University

\section{Yun Chen}

Second Xiangya Hospital of Central South University

\section{Yue Guo}

Second Xiangya Hospital of Central South University

Yun-Zhi Feng ( $\nabla$ fengyunzhi001@csu.edu.cn )

Second Xiangya Hospital of Central South University

\section{Research Article}

Keywords: infectious skin disease, leprosy, RNA-seq, CLOCK proteins, bone formation

Posted Date: August 5th, 2021

DOI: https://doi.org/10.21203/rs.3.rs-745191/v1

License: (c) (7) This work is licensed under a Creative Commons Attribution 4.0 International License. Read Full License 


\section{Abstract}

Bone formation and loss are the characteristic clinical manifestations of leprosy, but the mechanisms underlying the bone remodeling with Mycobacterium leprae (M. leprae) infection are unclear. Osteocytes may have a role through regulating the differentiation of osteogenic lineages. To investigate osteocyte-related mechanisms in leprosy, we treated osteocyte-like cell with N-glycosylated muramyl dipeptide (N.g MDP). RNA-seq analysis showed 724 differentially expressed messenger RNAs (mRNAs) and 724 differentially expressed circular RNA (circRNAs). Of these, we filtered through eight osteogenic-related differentially expressed genes, according to the characteristic of competing endogenous RNA, PubMed databases, and bioinformation analysis, including TargetScan, Gene Ontology, and Kyoto Encyclopedia of Genes and Genomes. Based on these results, we built a circRNA-microRNA (miRNA)-mRNA triple network. Quantitative reverse-transcription polymerase chain reaction and western blots analyses confirmed decreased Clock expression in osteocyte-like cell, while increased in bone mesenchymal stem cells (BMSCs), implicating a crucial factor in osteogenic differentiation. Immunohistochemistry showed obviously increased expression of CLOCK protein in BMSCs and osteoblasts in N.g MDP-treated mice, but decreased expression in osteocytes. This analytical method provided a basis for the relationship between N.g MDP and remodeling in osteocytes, and the circRNA-miRNA-mRNA triple network may offer a new target for leprosy therapeutics.

\section{Introduction}

Leprosy, caused by the bacillus Mycobacterium leprae ( $M$. leprae), is a chronic infectious disease, with more than 200,000 cases occurring annually worldwide ${ }^{1}$. Previous studies have shown that these patients are at risk for being misdiagnosed with other diseases, such as non-specified connective tissue disease and nodular vasculitis, delaying appropriate treatment ${ }^{2}$. The clinical manifestations of leprosy include lesions of the skin and peripheral nerves ${ }^{3}$ and deformity and physical disability ${ }^{4}$. Poor oral hygiene also has been noted ${ }^{5,6}$. The mechanism underlying these clinical manifestations remain to be elaborated, however.

Leprosy leads to bone changes, but what causes these changes is controversial. Some researchers have suggested bone destruction as a likely explanation, and indeed, bone damage is observed in $90 \%$ of patients ${ }^{7}$. Furthermore, $40 \%$ of patients show absorption of terminal phalanges on radiology ${ }^{8}$, resulting from the expression of a phosphateregulating gene with homologies to endopeptidase on the $\mathrm{X}$ chromosome (PHEX) that facilitates suppression by $M$. leprae in osteoblasts ${ }^{9}$. Other authors, however, have reported finding bone formation in patients with leprosy ${ }^{10}$. Tolllike receptor (TLR) 1 and TLR4 polymorphisms are associated with protection against leprosy ${ }^{11,12}$, and mineralizationrelated protein is elevated in the condition ${ }^{13}$. Bone islands and osteosclerosis also have been identified by radiology in these patients ${ }^{14,15}$. Thus, the pathways leading to bone alteration in leprosy remain unclear.

Culturing M. leprae in vitro could crucially contribute to understanding of the bone remodeling mechanism, but this species cannot be cultured in artificial medium ${ }^{16}$. M. tuberculosis and M. leprae show phylogenetic proximity ${ }^{17}$, and their bacterial products have been used in experimental analyses. Of these, muramyl dipeptide (MDP) is the minimal essential structure for the immunological effects of the cell wall ${ }^{16}$ and can occur as $\mathrm{N}$-acetyl MDP and N-glycosylated MDP (N.g MDP). N.g MDP is known to trigger an exceptionally strong immunogenic response ${ }^{18}$.

Osteogenic lineages are key in modulating bone formation, and osteocytes especially play a central role in bone homeostasis $^{19}$. Osteocytes produce and secrete molecules such as Dickkopf-1, fibroblast growth factor 23 , dentine matrix protein-1, PHEX, and matrix extracellular phosphoglycoprotein to mediate osteoblast differentiation, viability, and function ${ }^{20-22}$. In high-glucose conditions, osteocyte-derived exosomes carrying miR-124-3p can regulate Gal-3 
expression of osteoblasts ${ }^{23}$, and overexpression of Col1a2 in the osteocytes of insulin-receptor substrate-1-deficient mice (Irs-1 ${ }^{-/-}$) can promote bone formation ${ }^{24}$.

A large class of non-coding RNAs (ncRNAs) has been discovered through large-scale genome and transcriptome studies in recent years ${ }^{25}$, including microRNAs (miRNAs) and circular RNAs (circRNAs). The miRNAs consist of $~ 22$

nucleotides and have a crucial role in gene silencing ${ }^{26}$. Jorge et al. reported that miR-101, miR-196b, miR-27b, and miR29 c serve as biomarkers in the diagnosis of a subtype of leprosy ${ }^{27}$, and Liu et al. found that through miRNA-21 expression, the host cell can ward off infection by $M$. leprae, preventing bacillus growth, generating natural barriers, and regulating the antibacterial pathway ${ }^{28}$. In addition, miR-342 mediates regulation of Col1a2 expression in bone formation ${ }^{24}$. The circRNAs may have potentially important roles in gene regulation, as well ${ }^{29}$. These ncRNAs are produced through a downstream splice-donor site covalently linked to an upstream splice-acceptor site, in a process called backsplicing ${ }^{30}$. CircRNAs contain many miRNAs response elements and competing binding sites, thereby reducing miRNAs and mRNAs interaction and regulating the expression of target genes at the posttranscriptional level. Thus, it is more likely to have competing endogenous (ce)RNA function and act as miRNA sponges to restrain the expression of miRNA and regulate RNA transcription. ${ }^{30-32}$ The circRNA Hsa_circ_0074834 has been associated with repair of bone defects and promotion of bone mesenchymal stem cells (BMSCs) osteogenic differentiation by acting as a ceRNA for miR-942-5 $p^{33}$. However, the involvement of circRNA in leprosy is unclear.

For a better understanding of gene expression in leprosy, the potential circRNA-miRNA-mRNA triple network needs to be investigated because it represents a possible therapeutic target. In this study, we constructed this triple network, using murine osteocyte-like MLO-Y4 cells treated with N.g MDP to elucidate the activity of this pathway in leprosy.

\section{Results}

\section{Differentially expressed mRNAs and circRNAs in N.g MDP-treated osteocyte-like cell}

Osteocyte-like cell was observed under a light microscope, and western blots showed dentine matrix protein 1 (DMP-1) expression in osteocyte-like cell, while not be detected in MC3T3-E1 (Supplemental figure 1). With the cutoff criteria of a fold-change $>1.5$ and $P<0.05$, we identified 724 differentially expressed mRNAs and circRNAs between control and N.g MDP-treated samples, with 579 up-regulated and 145 down-regulated genes in differentially expressed mRNAs and 309 up-regulated and 415 down-regulated circRNAs (Figure 1). The top-10 dysregulated mRNAs (Table 1) and circRNAs (Table 2) were summarized based on P values. Bioinformatics analysis of the interactions (Table 3 ) and gene pathways between the two samples have been submitted. Search Tool for the Retrieval of Interacting Genes (STRING) database analysis identified 717 nodes and 1218 edges (Supplemental figure 2).

\section{Construction of the circRNA-miRNA-mRNA triple network}

To investigate ceRNA regulation in N.g MDP-treated osteocyte-like cell and characterize the osteoblastic-related circRNA-miRNA-mRNA triple network, we used TargetScan (http://www.targetscan.org/) databases to predict miRNA-mRNA and obtained 1912 miRNA-mRNA interaction pairs (data not shown). Using the circRNA database provided by GuangZhou Forevergen Biosciences Co., Ltd., we obtained 3307 circRNA-miRNA interaction pairs. Furthermore, we screened out the expression of mRNA and circRNA, which were consistent. Using the cutoff criteria of total context++ score $<-0.4$ and aggregate probability of conserved targeting $>0.4$ in the TargetScan software, we obtained 58 circRNA-miRNA-mRNA interaction pairs (Figure 2). We first selected mRNAs with expression meeting a fold-change threshold $>2$ and identified 14 differentially expressed genes (Dbndd1, Rcan2, Kif24, Cacnb4, Atf7ip2, Lin28b, 5031414D18Rik, Npnt, Lpar6, Myb/1, Ttc14, Zc3h12b, Plag1, and Alg6). In related searches of PubMed, we 
found six that were osteoblastic-related (Rcan2, Cacnb4, Plag1, Lpar6, Npnt, and Lin28b). We also assessed a selection of 58 mRNAs by Gene Ontology (GO) (Table 4) and Kyoto Encyclopedia of Genes and Genomes (KEGG) analysis (Figures 3 and 4A, B). Bioinformatics analysis of gene pathways identified "Circadian rhythm" as uniquely associated with the differentially expressed mRNA, including Clock and Rora.

\section{De-regulation of Clock expression in N.g MDP-treated osteocyte-like cell in vitro and in femurs of N.g MDP-treated mice in vivo}

To verify in vitro and in vivo the accuracy of the filtered results for the differentially expressed mRNAs,

osteocyte-like cell were treated with or without (control) $1 \mu \mathrm{g} / \mathrm{ml} \mathrm{N.g} \mathrm{MDP} \mathrm{for} 36 \mathrm{~h}$, and quantitative reversetranscription polymerase chain reaction (qRT-PCR) was used to measure and compare the expression of the identified genes in control and treated samples. We found that the expression of ClockmRNA was decreased approximately $20 \%$ $(P<0.05)$, but found no other significant differences between the two samples (Figure 4C).

Western blots showed an obviously decreasing amount of CLOCK protein $(P<0.05)$ (Figure 4D and E), along with a tendency for expression of the osteogenic differentiation biomarkers Runx2 and Bglap to decline. The qRT-PCR showed that Clock mRNA was upregulated in BMSCs under osteogenic induction (Figure 4F). To illustrate the expression of CLOCK protein in vivo, mice were infused with $2 \mu \mathrm{g} \mathrm{N.g} \mathrm{MDP} \mathrm{dissolved} \mathrm{in} 100 \mu \mathrm{l}$ of saline solution once a day for 10 days, and the control group was infused with $100 \mu$ saline only. Immunohistochemistry showed increased CLOCK protein expression in BMSCs and osteoblasts from femurs of N.g MDP-treated animals, but decreased expression in osteocytes (Figure 4G-I).

\section{Discussion}

In this study, we analyzed RNA-seq results of mRNA and circRNA, and integrated these findings with bioinformatics analysis to construct a circRNA-miRNA-mRNA triple network. According to the two different analytical methods we applied, N.g MDP may control the process of osteoblastic differentiation through regulation of Clock expression in osteocyte-like cell, suggesting a potential therapeutic target in leprosy-related bone loss.

In the context of bone metabolism, RNA-seq has influenced every aspect of understanding of genomic function ${ }^{34,35}$. Using a combination of large-scale genome and transcriptome studies with bioinformation analysis, Xu and colleagues found that targeting the SLIT3 pathway could represent a new approach to treating bone loss ${ }^{36}$. However, no related study has demonstrated an interaction between ncRNA and leprosy. As noted, $M$. leprae cannot be cultured in vitro, representing an obstacle to researching bone remodeling mechanisms in this disease ${ }^{16,37,38}$.

MDP is a component of the cell wall and the minimal essential structure for eliciting immunological effects ${ }^{16}$. To further investigate the relationship between MDP and ncRNA, we developed an in vitro MDP-treated osteocyte-like cell model and identified several differentially expressed mRNAs and circRNAs. However, because we could not verify all of these differentially expressed genes in experimental analysis, we used TargetScan and the circRNA database (provided by GuangZhou Forevergen Biosciences Co., Ltd.) to predict the target miRNA and construct a circRNA-miRNA-mRNA triple network. The circRNA-miRNA-mRNA triple network was wildly applied in research, Chen $\mathrm{X}$ et al. found that CircRNA_28313/miR-195a/CSF1 could regulate osteoclastic differentiation in OVX-induced bone absorption in mice $^{39}$, and Shen WX et al. revealed that CircFOXP1/miR-33a-5p/FOXP1 could promote osteogenic differentiation and CircFOXP1 can be used as a potential osteoporosis therapeutic target ${ }^{40}$. Based on the predicted results, we screened out the differentially expressed genes using a fold-change cutoff of $>2.0$ for $m R N A^{41}$. We narrowed the list to six differentially expressed mRNAs using a PubMed search and to two differentially expressed mRNAs using GO and 
KEGG analysis. We confirmed decreased expression of the Clock gene, one of the two identified with the latter approach, but that expression of Rcan2, Cacnb4, Plag1, Lpar6, Npnt, Lin28b, and Rora did not differ between treated and untreated samples (Fig. 4C). Thus, this circRNA-miRNA-mRNA triple network could provide a new analytical approach to elucidating the relationship between N.g MDP and bone remodeling.

The circadian clock is a key factor in the circadian system, which is associated with cell function, metabolic state, and life expectancy ${ }^{42,43}$. Bone remodeling could be subject to circadian regulation ${ }^{44}$, and a recent study demonstrated that the circadian clock could regulate bone formation through transcriptional control of the 1,25(OH)2D3 receptor PDIA3 ${ }^{45}$. Decreasing of the bone mineral density and increasing risk of patients developing osteoporosis would happen, if the circadian rhythm has disordered ${ }^{46}$. Besides, the core clock gene, brain and muscle Arnt -like protein 1 (BMAL1), could influence numbers of key factors in skeletal development, such as Runx2/Osterix and HIF1a-VEGF signaling pathway ${ }^{47}$. Our results showed decreased expression of Clock, along with expression of the osteogenic biomarkers Runx2 and Bglap (Fig. 4D), while the expression of Clock was increased in BMSCs under osteogenic induction. Yuan $\mathrm{HP}$ et al. found that intraperitoneal injection of $\mathrm{MDP}$ in $\mathrm{ApoE}^{-/-}$mice could reduce alveolar bone loss which expose in porphyromonas gingivalis ${ }^{48}$. Park $\mathrm{OJ}$ et al. revealed that MDP could induce bone formation and osteoblast activation by upregulating the expression of Runx2 and ALP in BMSCs and preosteoblasts, and micro-CT showed increased trabecular bone in the femur of MDP-administered mice ${ }^{49}$. However, the regulatory effect of MDP on osteocyte still not be reported. Immunohistochemical analysis indicated that expression of the CLOCK protein was significantly increased in murine femur BMSCs and osteoblasts, while decreased in osteocytes in exposed mice (Fig. 4E). The paradoxical results possibly because of negative feedback regulation of osteocytes to osteoblasts and $\mathrm{BMSCs}^{50}$, and indicate that different cells induce different responses in vivo and in vitro. In general, these results suggest a crucial role for Clock in bone remodeling.

In summary, based on the results of transcriptome studies and bioinformation analysis, we have characterized a circRNA-miRNA-mRNA triple network and screened out the key factors in the N.g MDP-treated osteocyte-like cell. Our approach potentially represents a new analytical method for elucidating the mechanism of bone remodeling in leprosy.

\section{Materials And Methods}

\section{Cell culture and treatment}

MLO-Y4 cells, a murine osteocyte-like cell line, MC3T3-E1, a murine preosteoblast cell, were purchased from the Cell Bank of the Chinese Academy of Sciences (Shanghai, China). Cells were maintained at a density of $2.5 \times 10^{6} /$ well in 6 well plates kept in a humidified incubator at $5 \% \mathrm{CO}_{2}$ at $37^{\circ} \mathrm{C}$. Culture medium consisted of a-modified minimum essential medium (Gibco, Thermo Fisher Scientific, Inc., Waltham, MA, USA), supplemented with 1\% Glutamax, 0.5\% mycoplasma antibiotics, 10\% fetal bovine serum (Gibco), and 1\% penicillin and streptomycin. For the treated group, 1 $\mu \mathrm{g} / \mathrm{ml} \mathrm{N}-\mathrm{g}$ MDP (Catalogue tIrl-gmdp, Invivogen) was applied for $36 \mathrm{~h}$. Both BMSCs preparation and osteogenic induction have been reported before ${ }^{24}$. Briefly, BMSCs were cultured in osteogenic medium, which involved $10 \mathrm{mM} \beta-$ glycerophosphate, $10^{-8} \mathrm{M}$ dexamethasone and $50 \mu \mathrm{g} / \mathrm{ml} \mathrm{I-2-ascorbic} \mathrm{acid.}$

\section{Animals}

All protocols used in our animal experiments were approved by the Animal Ethics Committee of the Central South University and conformed with the ARRIVE guidelines. All methods were performed in accordance with the relevant guidelines and regulations. The C57BL/ 6 female mice were supplied by the Experimental Animal Center of Xiang-Ya Second Hospital. After a week of adjustable feeding, mice in the exposure group were infused with $2 \mu \mathrm{g}$ N.g MDP 
dissolved in $100 \mu \mathrm{l}$ of saline solution once a day for 10 days, and the control group was infused with $10 \mu \mathrm{l}$ saline only. Both groups were euthanized with an overdose of anesthesia (pentobarbital sodium). We have previously published the details of femur removal ${ }^{24}$. Investigators were blinded to the group allocation during the experiment and when assessing the outcome.

\section{mRNA/circRNA sequencing}

Total RNA was extracted from the samples in Trizol reagent according to the manufacturer's instructions (Invitrogen). The concentration and purity of each RNA sample were determined using the dsDNA HS Assay kit for Qubit (12640ES76, Yeasen). The quality of the library was determined using an Agilent High Sensitivity DNA Kit (5067-4626, Agilent), and integrity and size were quantified with this kit on an Agilent 2100 Bioanalyser (Agilent Technologies, Santa Clara, CA). For the mRNA library, mRNA was purified via two rounds of hybridization to Dynal Oligo beads (N411-03, Vazyme). After depleting the samples of ribosomal RNAs, we applied fragmentation buffer (AM8740; Invitrogen) and synthesized cDNA from the fragments using random primers. Following end repair second-strand digestion and adaptor ligation, the purified fragments were PCR amplified. For the circRNA library, total RNA was subjected to ribosomal RNA depletion using the QIAseq FastSelect RNA Removal Kit (333180, QIAGEN). Remaining RNA samples were treated with RNase R (RNR07250; Epicenter) to remove linear RNAs. After preparation of cDNA, the remaining procedures were similar to those for the mRNA library. The library preparation was performed using the VAHTS mRNA-seq V3 Library Prep Kit for Illumina (NR611, Vazyme).

\section{Bioinformatics analysis}

TargetScan (http://www.targetscan.org/) software packages were used to predict the potential miRNA targets of the mRNAs. The biological processes involving these genes and KEGG (adjusted P value < 0.05 ; gene count $\geq 2$ ) were obtained from the database for Annotation, Visualization, and Integrated Discovery (known as DAVID) (https://david.ncifcrf.gov/) (gene count $\geq 2$ ). The differentially expressed mRNAs and proteins were visualized using STRING software (https://string-db.org/). The circRNA-miRNA-mRNA interaction network was built by merging the circRNA database (providing by GuangZhou Forevergen Biosciences), differentially expressed genes, and the predicted potential miRNA.

\section{qRT-PCR}

After extraction, RNA $(1 \mu \mathrm{g})$ was reverse-transcribed (PrimeScript RT kit; TaKaRa, Otsu, Japan) and qPCR performed (SYBR Premix Ex Taq II kit; TaKaRa) using the following primer sequences: Rcan2 forward, 5'ACCTATGATGAATGTGTGACGT-3', reverse, 5' -TTAGCTTCTTCCCTCTGAACTG-3'; Cacnb4 forward, 5'GTTTTACAGCGGCTGATTAAGT-3', reverse, 5' -TAACATCAAACATTTCAGGCGG-3'; Plag1 forward, 5'CTTTCAGTGGGAAGCCTTGGGATG-3', reverse, 5' -GAACGCTGCCGACAGTGAGTG-3'; Lpar6 forward, 5'ATCGTTTGCATTGCTGTGTGGTTC-3', reverse, 5' -GCAGGCTTCTGAGGTATTGTTCCC-3'; Npnt forward, 5'GGTGATGGAGGACATGCGAATAGG-3', reverse, 5' -GTAGGCTGTGGTGTTGGGTTTGG-3'; Lin28b forward, 5'GGAGACGGCAGGATTTACTGATGG-3', reverse, 5' -AATGGCACTTCTTTGGCTGAGGAG-3'; Clock forward, 5'TTCCTGACCAAAGGCCAGCA-3', reverse, 5'- CTCGCCGTCTTTCAGCCCTA-3'; Rora forward, 5'-

\section{TGGTGGAGTTTGCCAAACGC}

-3', reverse, 5'- TGAGAGTCAAAGGCACGGCA-3'; and $\beta$-actin forward, 5'-CAACGAGCGGTTCCGATG-3', reverse, 5'GCCACAGGATTCCATACCCA-3' (all Shanghai Generay Biotech Co., Ltd., Shanghai, China). Cycling conditions for PCR (LightCycler Real-time PCR System, Roche) were as follows: $30 \mathrm{~s}$ at $95^{\circ} \mathrm{C}$ for polymerase activation; 40 cycles of $5 \mathrm{~s}$ 
each at $95^{\circ} \mathrm{C}$ and of $20 \mathrm{~s}$ each at $60^{\circ} \mathrm{C}$. Primer specificity was confirmed using melting curves, and the 2- $\Delta \Delta \mathrm{Ct}$ method was used to analyze the qRT-PCR results.

\section{Western blots}

Western blots were performed as previously described ${ }^{23}$. Briefly, total protein extracts were prepared in RIPA buffer, which contained inhibitors of proteases and phosphatases. After electrophoresis, the SDS-PAGE-separated proteins were transferred to a polyvinylidene fluoride membrane (EMD-Millipore, Billerica, MA, USA). The membranes were blocked with QuickBlock (P0252, beyotime) for 20 min and incubated overnight at $4{ }^{\circ} \mathrm{C}$ in $3 \%$ bovine serum albumin (9048-46-8, Sigma-Aldrich) in TBST supplemented with primary antibodies against CLOCK (AF0323, Affinity Biosciences), RUNX2 (ab23981, Abcam), Osteocalcin (PA5-86886, Invitrogen), and $\beta$-actin (AM1021B, Abcepta). Immunoreactive bands were detected using an antirabbit peroxidase-conjugated secondary antibody (1:5000; Bioss) and visualized with enhanced chemiluminescence (GE Healthcare, Little Chalfont, UK).

\section{Immunohistochemistry}

Immunohistochemistry was performed as previously described ${ }^{23}$. Briefly, right femurs were fixed in $4 \%$ paraformaldehyde for 24-48 h, decalcified in 10\% EDTA for $21 \mathrm{~d}$, and embedded in paraffin. Decalcified right femur sections $5 \mu \mathrm{m}$ thick were deparaffinized in turpentine, $3 \% \mathrm{H}_{2} \mathrm{O}_{2}$ was used to suppress endogenous peroxidase activity, and the sections were treated with $0.1 \%$ trypsin for antigen retrieval. After incubation with a CLOCK polyclonal antibody (AF0323; 1:100, Affinity Biosciences) overnight, sections were incubated with secondary antibodies. Antibodies were detected by staining with a horseradish peroxidase-conjugated rabbit anti-mouse IgG and diaminobenzidine (GTVision III Detection System/Mo\&Rb Kit; Gene Tech, Shanghai, China). Specimens were counterstained with hematoxylin.

\section{Statistical analysis}

All experiments were carried out in triplicate. Data were analyzed using IBM SPSS Statistics 26 . Statistical significance was considered at $\mathrm{P}<0.05$ using the Student's t-test. The results are presented as mean \pm standard deviation.

\section{Declarations}

\section{Acknowledgments}

This study was supported by the National Natural Science Foundation of China (Grants 81773339 and 81800788), the Science and Technology Department of Hunan Province, China (Grants 2017WK2041 and 2018SK52511), the fund for Xiangya Clinical Medicine Database of Central South University (Grant 2014-ZDYZ-1-16), and the Open Sharing Fund for the Large-scale Instruments and Equipment of Central South University.

\section{Contributorship statement}

Yue Guo and Yun-Zhi Feng designed and conducted of the study; Zheng-Rong Gao and Qiong Liu provided the data and performed the research; Zheng-Rong Gao, Jie Zhao and Ya-Qiong Zhao analyzed and interpreted the data; Li Tan and Shao-Hui Zhang developed software necessary to perform and record experiments. Ying-Hui Zhou and Yun Chen performed research and revised the manuscript; and Zheng-Rong Gao wrote the paper.

\section{Conflict of Interest Statement}

All authors declare no conflicts of interest. 


\section{Data Availability Statement}

The data used to support the findings of this study are available from the corresponding author upon request.

\section{References}

1. Pandey, A. \& Galvani, A. Quantifying risk factors to guide progress towards leprosy elimination. The Lancet. Global health 7, e1154-e1155, doi:10.1016/s2214-109x(19)30330-4 (2019).

2. Zhang, G., Jin, H., Chen, H. \& Lu, Q. Cutaneous nodules with positive autoantibodies: histoid leprosy. Lancet (London, England) 386, 1915-1916, doi:10.1016/s0140-6736(15)00678-9 (2015).

3. Smith, C. et al. A strategy to halt leprosy transmission. The Lancet. Infectious diseases 14, 96-98, doi:10.1016/s1473-3099(13)70365-7 (2014).

4. Netea, M., Kullberg, B. \& van der Meer, J. Genomewide association study of leprosy. The New England journal of medicine 362, 1447; author reply 1447-1448 (2010).

5. Guo, Y. et al. Dental caries and risk indicators for patients with leprosy in China. Int. Dent. J. 67, 59-64, doi:10.1111/idj.12257 (2017).

6. Feng, Y. et al. Dental health and treatment needs in people with leprosy in China. Lepr. Rev. 85, 311-321 (2014).

7. Mohammad, W., Malhotra, S. \& Garg, P. Clinico-radiological Correlation of Bone Changes in Leprosy Patients Presenting with Disabilities/Deformities. Indian journal of leprosy 88, 83-95 (2016).

8. Silva, S. et al. Downregulation of PHEX in multibacillary leprosy patients: observational cross-sectional study. J. Transl. Med. 13, 296, doi:10.1186/s12967-015-0651-5 (2015).

9. Silva, S. et al. Mycobacterium leprae downregulates the expression of PHEX in Schwann cells and osteoblasts. Mem. Inst. Oswaldo Cruz 105, 627-632, doi:10.1590/s0074-02762010000500005 (2010).

10. González-Reimers, E. et al. A skeletal case of hypertrophic osteoarthropathy from the Canary Islands dating from 1000 BP. Int J Paleopathol 11, 1-6, doi:10.1016/j.ijpp.2015.07.001 (2015).

11. Misch, E. et al. Human TLR1 deficiency is associated with impaired mycobacterial signaling and protection from leprosy reversal reaction. PLoS Negl. Trop. Dis. 2, e231, doi:10.1371/journal.pntd.0000231 (2008).

12. Bochud, P. et al. Polymorphisms in Toll-like receptor 4 (TLR4) are associated with protection against leprosy. European journal of clinical microbiology \& infectious diseases : official publication of the European Society of Clinical Microbiology 28, 1055-1065, doi:10.1007/s10096-009-0746-0 (2009).

13. Hoffman, V. \& Korzeniowski, O. Leprosy, hypercalcemia, and elevated serum calcitriol levels. Ann. Intern. Med. 105, 890-891, doi:10.7326/0003-4819-105-6-890 (1986).

14. Simone, C. \& Racanelli, A. Osteosclerosis in leprosy. Italian journal of orthopaedics and traumatology 8, 211-219 (1982).

15. Carpintero, P. et al. Bone island and leprosy. Skeletal Radiol. 27, 330-333, doi:10.1007/s002560050391 (1998).

16. Behr, M. A. \& Divangahi, M. Freund's adjuvant, NOD2 and mycobacteria. Curr. Opin. Microbiol. 23, 126-132, doi:10.1016/j.mib.2014.11.015 (2015).

17. B, C.-P., CY, S. \& MI, G. Mycobacterium leprae's evolution and environmental adaptation. Acta Trop. 197, 105041, doi:10.1016/j.actatropica.2019.105041 (2019).

18. F, C. et al. Increased NOD2-mediated recognition of N-glycolyl muramyl dipeptide. The Journal of experimental medicine 206, 1709-1716, doi:10.1084/jem.20081779 (2009).

19. Hayashi, M. et al. Autoregulation of Osteocyte Sema3A Orchestrates Estrogen Action and Counteracts Bone Aging. Cell Metab. 29, 627-637.e625, doi:10.1016/j.cmet.2018.12.021 (2019). 
20. Heiland, G. et al. Neutralisation of Dkk-1 protects from systemic bone loss during inflammation and reduces sclerostin expression. Ann. Rheum. Dis. 69, 2152-2159, doi:10.1136/ard.2010.132852 (2010).

21. Plotkin, L. I. \& Bellido, T. Osteocytic signalling pathways as therapeutic targets for bone fragility. Nature reviews. Endocrinology 12, 593-605, doi:10.1038/nrendo.2016.71 (2016).

22. Dussold, C. et al. DMP1 prevents osteocyte alterations, FGF23 elevation and left ventricular hypertrophy in mice with chronic kidney disease. \#N/A 7, 12, doi:10.1038/s41413-019-0051-1 (2019).

23. Li, J. et al. miR-124-3p increases in high glucose induced osteocyte-derived exosomes and regulates galectin-3 expression: A possible mechanism in bone remodeling alteration in diabetic periodontitis. FASEB journal : official publication of the Federation of American Societies for Experimental Biology 34, 14234-14249, doi:10.1096/fj.202000970RR (2020).

24. Guo, Y. et al. Insulin receptor substrate-1 time-dependently regulates bone formation by controlling collagen la2 expression via miR-342. FASEB journal : official publication of the Federation of American Societies for Experimental Biology 30, 4214-4226, doi:10.1096/fj.201600445RR (2016).

25. Zhang, F. et al. Comprehensive analysis of circRNA expression pattern and circRNA-miRNA-mRNA network in the pathogenesis of atherosclerosis in rabbits. Aging 10, 2266-2283, doi:10.18632/aging.101541 (2018).

26. Gebert, L. \& MacRae, I. Regulation of microRNA function in animals. Nature reviews. Molecular cell biology 20, 2137, doi:10.1038/s41580-018-0045-7 (2019).

27. Jorge, K. et al. Characterization of MicroRNA Expression Profiles and Identification of Potential Biomarkers in Leprosy. J. Clin. Microbiol. 55, 1516-1525, doi:10.1128/jcm.02408-16 (2017).

28. Liu, P. et al. MicroRNA-21 targets the vitamin D-dependent antimicrobial pathway in leprosy. Nat. Med. 18, 267-273, doi:10.1038/nm.2584 (2012).

29. Chen, L. The biogenesis and emerging roles of circular RNAs. Nature reviews. Molecular cell biology 17, 205-211, doi:10.1038/nrm.2015.32 (2016).

30. Kristensen, L. et al. The biogenesis, biology and characterization of circular RNAs. Nature reviews. Genetics 20, 675-691, doi:10.1038/s41576-019-0158-7 (2019).

31. Chen, L. The expanding regulatory mechanisms and cellular functions of circular RNAs. Nature reviews. Molecular cell biology 21, 475-490, doi:10.1038/s41580-020-0243-y (2020).

32. Jiao, J. et al. Development of a two-in-one integrated assay for the analysis of circRNA-microRNA interactions. \#N/A 178, 113032, doi:10.1016/j.bios.2021.113032 (2021).

33. Ouyang, Z. et al. CircRNA hsa_circ_0074834 promotes the osteogenesis-angiogenesis coupling process in bone mesenchymal stem cells (BMSCs) by acting as a ceRNA for miR-942-5p. Cell Death Dis. 10, 932, doi:10.1038/s41419-019-2161-5 (2019).

34. Tikhonova, A. et al. The bone marrow microenvironment at single-cell resolution. Nature 569, 222-228, doi:10.1038/s41586-019-1104-8 (2019).

35. Stark, R., Grzelak, M. \& Hadfield, J. RNA sequencing: the teenage years. Nature reviews. Genetics 20, 631-656, doi:10.1038/s41576-019-0150-2 (2019).

36. Xu, R. et al. Targeting skeletal endothelium to ameliorate bone loss. Nat. Med. 24, 823-833, doi:10.1038/s41591018-0020-z (2018).

37. Zhang, F. et al. Genomewide association study of leprosy. The New England journal of medicine 361, 2609-2618, doi:10.1056/NEJMoa0903753 (2009).

38. Chavarro-Portillo, B., Soto, C. \& Guerrero, M. Mycobacterium leprae's evolution and environmental adaptation. Acta Trop. 197, 105041, doi:10.1016/j.actatropica.2019.105041 (2019). 
39. Chen, X. et al. CircRNA_28313/miR-195a/CSF1 axis modulates osteoclast differentiation to affect OVX-induced bone absorption in mice. RNA Biol. 16, 1249-1262, doi:10.1080/15476286.2019.1624470 (2019).

40. Shen, W. et al. CircFOXP1/FOXP1 promotes osteogenic differentiation in adipose-derived mesenchymal stem cells and bone regeneration in osteoporosis via miR-33a-5p. J. Cell. Mol. Med. 24, 12513-12524, doi:10.1111/jcmm.15792 (2020).

41. Wang, C. et al. RNA-Seq Based Transcriptome Analysis of Endothelial Differentiation of Bone Marrow Mesenchymal Stem Cells. European journal of vascular and endovascular surgery : the official journal of the European Society for Vascular Surgery 59, 834-842, doi:10.1016/j.ejvs.2019.11.003 (2020).

42. Peek, C., Ramsey, K., Marcheva, B. \& Bass, J. Nutrient sensing and the circadian clock. Trends in endocrinology and metabolism: TEM 23, 312-318, doi:10.1016/j.tem.2012.02.003 (2012).

43. Bass, J. \& Takahashi, J. Circadian integration of metabolism and energetics. Science (New York, N. Y.) 330, 13491354, doi:10.1126/science.1195027 (2010).

44. Fu, L., Patel, M., Bradley, A., Wagner, E. \& Karsenty, G. The molecular clock mediates leptin-regulated bone formation. Cell 122, 803-815, doi:10.1016/j.cell.2005.06.028 (2005).

45. Yuan, G. et al. The Circadian Gene Clock Regulates Bone Formation Via PDIA3. Journal of bone and mineral research : the official journal of the American Society for Bone and Mineral Research 32, 861-871, doi:10.1002/jbmr.3046 (2017).

46. Zhuo, H., Wang, Y. \& Zhao, Q. The Interaction between Bmal1 and Per2 in Mouse BMSC Osteogenic Differentiation. \#N/A 2018, 3407821, doi:10.1155/2018/3407821 (2018).

47. Chen, G. et al. The biological function of BMAL1 in skeleton development and disorders. Life Sci. 253, 117636, doi:10.1016/j.Ifs.2020.117636 (2020).

48. Sullivan, J., Belloir, J. J. D. \& immunology, c. Activation of an innate immune response in the schistosometransmitting snail Biomphalaria glabrata by specific bacterial PAMPs. 42, 256-260, doi:10.1016/j.dci.2013.09.016 (2014).

49. Park, O., Kim, J., Yang, J., Yun, C. \& Han, S. Muramyl Dipeptide, a Shared Structural Motif of Peptidoglycans, Is a Novel Inducer of Bone Formation through Induction of Runx2. Journal of bone and mineral research : the official journal of the American Society for Bone and Mineral Research 32, 1455-1468, doi:10.1002/jbmr.3137 (2017).

50. Hassan, M. et al. A network connecting Runx2, SATB2, and the miR-23a 27a 24-2 cluster regulates the osteoblast differentiation program. Proc. Natl. Acad. Sci. U. S. A. 107, 19879-19884, doi:10.1073/pnas.1007698107 (2010).

\section{Tables}

Table 1. Top 10 dysregulated mRNAs in MLO-Y4 cells treated with N.g MDP 


\begin{tabular}{lllll} 
Gene & Fold-change & Status & $P$ & FDR \\
\hline S100a10 & 0.648097 & Down & $2.15 \mathrm{E}-76$ & $4.43 \mathrm{E}-74$ \\
\hline Rpl39I & 0.657591 & Down & $7.93 \mathrm{E}-65$ & $1.42 \mathrm{E}-62$ \\
\hline Eif4a2 & 1.567235 & Up & $1.11 \mathrm{E}-53$ & $1.77 \mathrm{E}-51$ \\
\hline Rps27I & 0.644698 & Down & $1.62 \mathrm{E}-52$ & $2.55 \mathrm{E}-50$ \\
\hline Cox7c & 0.606489 & Down & $1.91 \mathrm{E}-51$ & $2.98 \mathrm{E}-49$ \\
\hline Atp5e & 0.607108 & Down & $1.57 \mathrm{E}-45$ & $2.32 \mathrm{E}-43$ \\
\hline Uqcrh & 0.619202 & Down & $1.12 \mathrm{E}-44$ & $1.60 \mathrm{E}-42$ \\
\hline Luc7l2 & 1.675182 & Up & $1.01 \mathrm{E}-35$ & $1.20 \mathrm{E}-33$ \\
\hline Nfat5 & 1.643251 & Up & $5.42 \mathrm{E}-34$ & $6.03 \mathrm{E}-32$ \\
\hline Cox6c & 0.628503 & Down & $7.00 \mathrm{E}-32$ & $7.49 \mathrm{E}-30$
\end{tabular}

Table 2. Top 10 dysregulated circRNAs in MLO-Y4 cells treated with N.g MDP

\begin{tabular}{|c|c|c|c|c|}
\hline circRNA_ID & circBase_ID & genelD & Status & $\mathrm{P}$ \\
\hline chr18:83124916|83126841 & mmu_circ_0000909 & Zfp516 & Down & $\begin{array}{l}4.87 \mathrm{E}- \\
89\end{array}$ \\
\hline chr11:108873006|108873262 & & U6,SNORA70,SCARNA4,7SK,snoR38,U3 & Down & $\begin{array}{l}1.13 \mathrm{E}- \\
85\end{array}$ \\
\hline chr16:11144101|11144327 & & U6,7SK,SNORA17,5S_rRNA,U1,Zc3h7a & Down & $\begin{array}{l}1.49 \mathrm{E}- \\
84\end{array}$ \\
\hline chr17:39985361|39985627 & & AY036118 & Down & $\begin{array}{l}5.71 \mathrm{E}- \\
70\end{array}$ \\
\hline chr5:137753102|137753461 & & Trip6 & Down & $\begin{array}{l}4.78 \mathrm{E}- \\
69\end{array}$ \\
\hline chr7:17468476|17468859 & mmu_circ_0001545 & Dact3 & Down & $\begin{array}{l}3.31 \mathrm{E}- \\
68\end{array}$ \\
\hline chr11:104285236|104286607 & mmu_circ_0000331 & 1700081L11Rik & Down & $\begin{array}{l}7.54 \mathrm{E}- \\
63\end{array}$ \\
\hline chr19:4784724|4788042 & mmu_circ_0000918 & Rbm14 & Down & $\begin{array}{l}9.89 \mathrm{E}- \\
63\end{array}$ \\
\hline chr3:51111972|51129957 & mmu_circ_0001125 & Elf2 & Up & $\begin{array}{l}3.83 \mathrm{E}- \\
59\end{array}$ \\
\hline chr16:15887401|15887572 & & Cebpd & Down & $\begin{array}{l}1.24 \mathrm{E}- \\
57\end{array}$ \\
\hline
\end{tabular}

Table 3. The significantly enriched pathways associated with 724 mRNAs in the N.g MDP-treated osteocyte model 


\begin{tabular}{|c|c|c|c|c|}
\hline Category & Pathway & Description & $\begin{array}{l}\text { Gene } \\
\text { count }\end{array}$ & $P$ \\
\hline \multirow{5}{*}{$\begin{array}{l}\text { Biological } \\
\text { process }\end{array}$} & Regulation of transcription, DNA-templated & GO:0006355 & 127 & 2.27E-09 \\
\hline & Ureteric bud development & G0:0001657 & 7 & 0.004772 \\
\hline & Transcription, DNA-templated & G0:0006351 & 82 & 0.007932 \\
\hline & Necroptotic process & GO:0070266 & 4 & 0.007997 \\
\hline & Inhibitory postsynaptic potential & GO:0060080 & 4 & 0.007997 \\
\hline \multirow{5}{*}{$\begin{array}{l}\text { Cellular } \\
\text { component }\end{array}$} & Intracellular & GO:0005622 & 94 & $2.07 \mathrm{E}-08$ \\
\hline & Ciliary transition zone & GO:0035869 & 7 & $2.31 \mathrm{E}-04$ \\
\hline & Nucleus & GO:0005634 & 224 & 0.008896 \\
\hline & $\begin{array}{l}\text { Mitochondrial proton-transporting ATP synthase } \\
\text { complex }\end{array}$ & GO:0005753 & 4 & 0.019214 \\
\hline & Endoplasmic reticulum & GO:0005783 & 56 & 0.033769 \\
\hline \multirow{5}{*}{$\begin{array}{l}\text { Molecular } \\
\text { function }\end{array}$} & Nucleic acid binding & GO:0003676 & 95 & $3.96 \mathrm{E}-14$ \\
\hline & Metal ion binding & GO:0046872 & 182 & 3.83E-12 \\
\hline & $\begin{array}{l}\text { Transcription factor activity, sequence-specific DNA } \\
\text { binding }\end{array}$ & GO:0003700 & 50 & $3.88 \mathrm{E}-04$ \\
\hline & DNA binding & G0:0003677 & 89 & 4.32E-04 \\
\hline & Syntaxin binding & GO:0019905 & 8 & 0.017731 \\
\hline
\end{tabular}

Table 4. The significantly enriched pathways associated with 58 mRNAs in the N.g MDP-treated osteocyte model 


\begin{tabular}{|c|c|c|c|c|}
\hline Category & Pathway & Description & $\begin{array}{l}\text { Gene } \\
\text { count }\end{array}$ & $P$ \\
\hline \multirow[t]{5}{*}{$\begin{array}{l}\text { Biological } \\
\text { process }\end{array}$} & $\begin{array}{l}\text { Positive regulation of transcription from RNA polymerase II } \\
\text { promoter }\end{array}$ & GO:0045944 & 9 & 0.004934 \\
\hline & Transcription, DNA-templated & G0:0006351 & 12 & 0.010709 \\
\hline & Regulation of transcription, DNA-templated & G0:0006355 & 13 & 0.016459 \\
\hline & Protein transport & G0:0015031 & 6 & 0.021561 \\
\hline & Intracellular protein transport & G0:0006886 & 4 & 0.024918 \\
\hline \multirow{3}{*}{$\begin{array}{l}\text { Cellular } \\
\text { component }\end{array}$} & Basement membrane & G0:0005604 & 3 & 0.027222 \\
\hline & Cytoplasm & G0:0005737 & 24 & 0.074427 \\
\hline & Membrane coat & G0:0030117 & 2 & 0.076426 \\
\hline \multirow[t]{5}{*}{$\begin{array}{l}\text { Molecular } \\
\text { function }\end{array}$} & $\begin{array}{l}\text { Transcriptional activator activity, RNA polymerase II core } \\
\text { promoter proximal region sequence-specific binding }\end{array}$ & GO:0001077 & 5 & 0.00508 \\
\hline & $\begin{array}{l}\text { RNA polymerase II core promoter proximal region sequence- } \\
\text { specific DNA binding }\end{array}$ & GO:0000978 & 5 & 0.01353 \\
\hline & DNA binding & G0:0003677 & 11 & 0.017334 \\
\hline & $\begin{array}{l}\text { RNA polymerase II transcription factor activity, sequence- } \\
\text { specific DNA binding }\end{array}$ & GO:0000981 & 3 & 0.074094 \\
\hline & Metal ion binding & GO:0046872 & 14 & 0.077303 \\
\hline
\end{tabular}

\section{Figures}


a

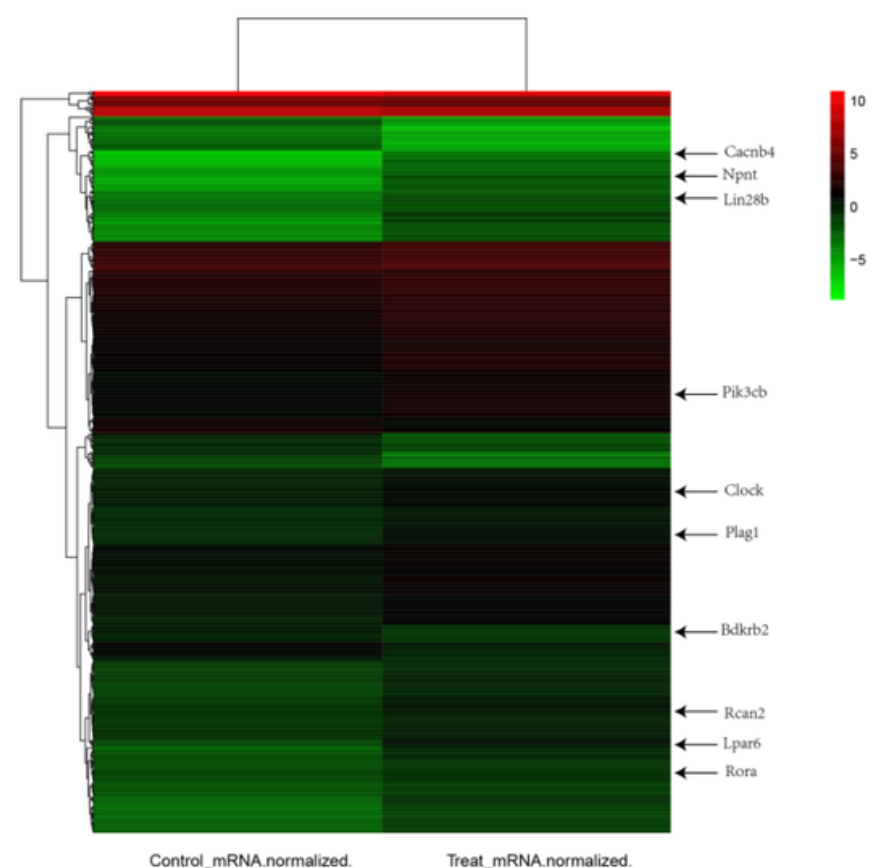

b

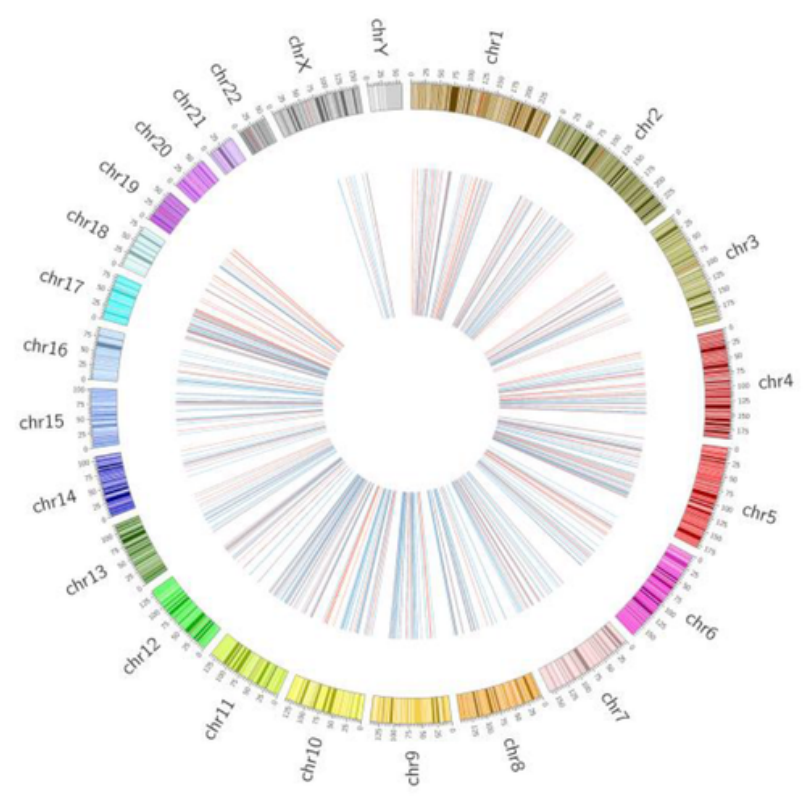

\section{Figure 1}

Identification of differentially expressed mRNAs and circRNAs. Heatmap of expression profiles of different mRNAs (a) and circRNAs (b), respectively (fold-change $>1.5$ and $P<0.05$ ). Red and green/blue denote high and low relative expression, respectively. 


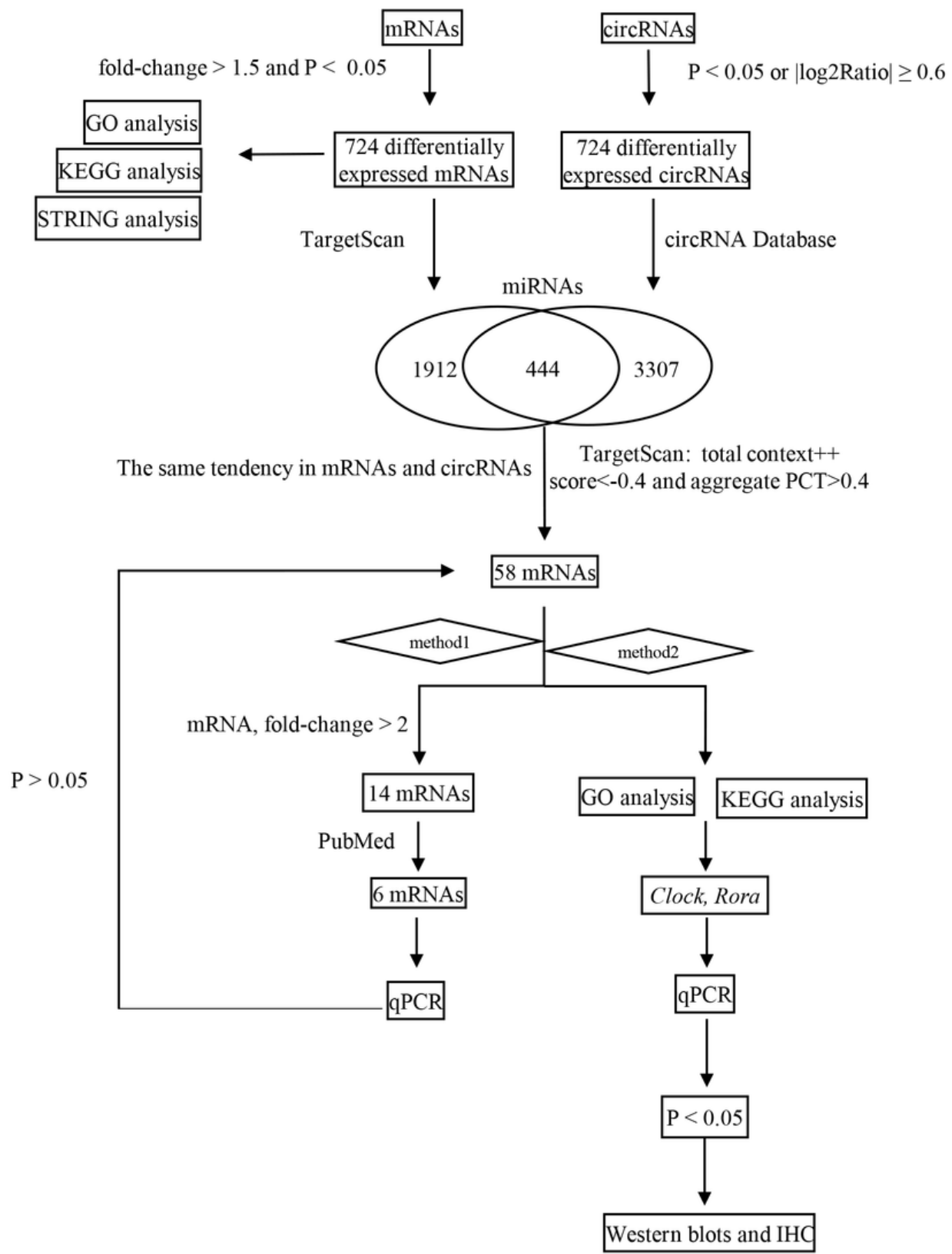

\section{Figure 2}

Combination of mRNA and circRNA databases to construct the circRNA-miRNA-mRNA triple network. 
a

Biological process

Cellular component

Molecular function

30

25

20

15

10

5

0
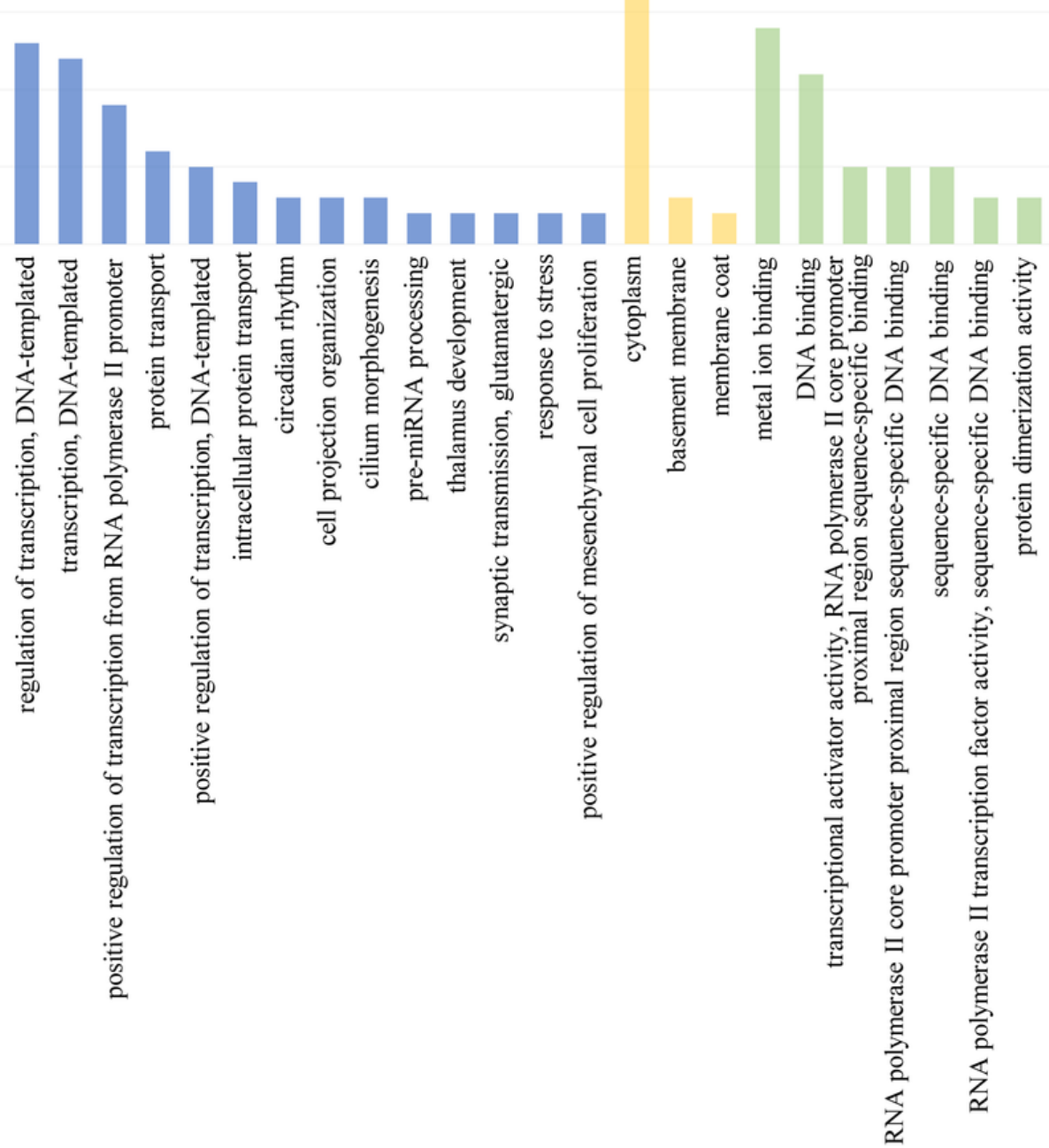

\section{Figure 3}

Bioinformatics analysis of the interactions among the differentially expressed genes in the N.g MDP-treated osteocytes. (a) The most significantly enriched GO (-log10 (P value)) terms of mRNA gene symbols according to biological process are marked in blue; cellular component in yellow; and molecular function in green. GO, gene ontology. 
A

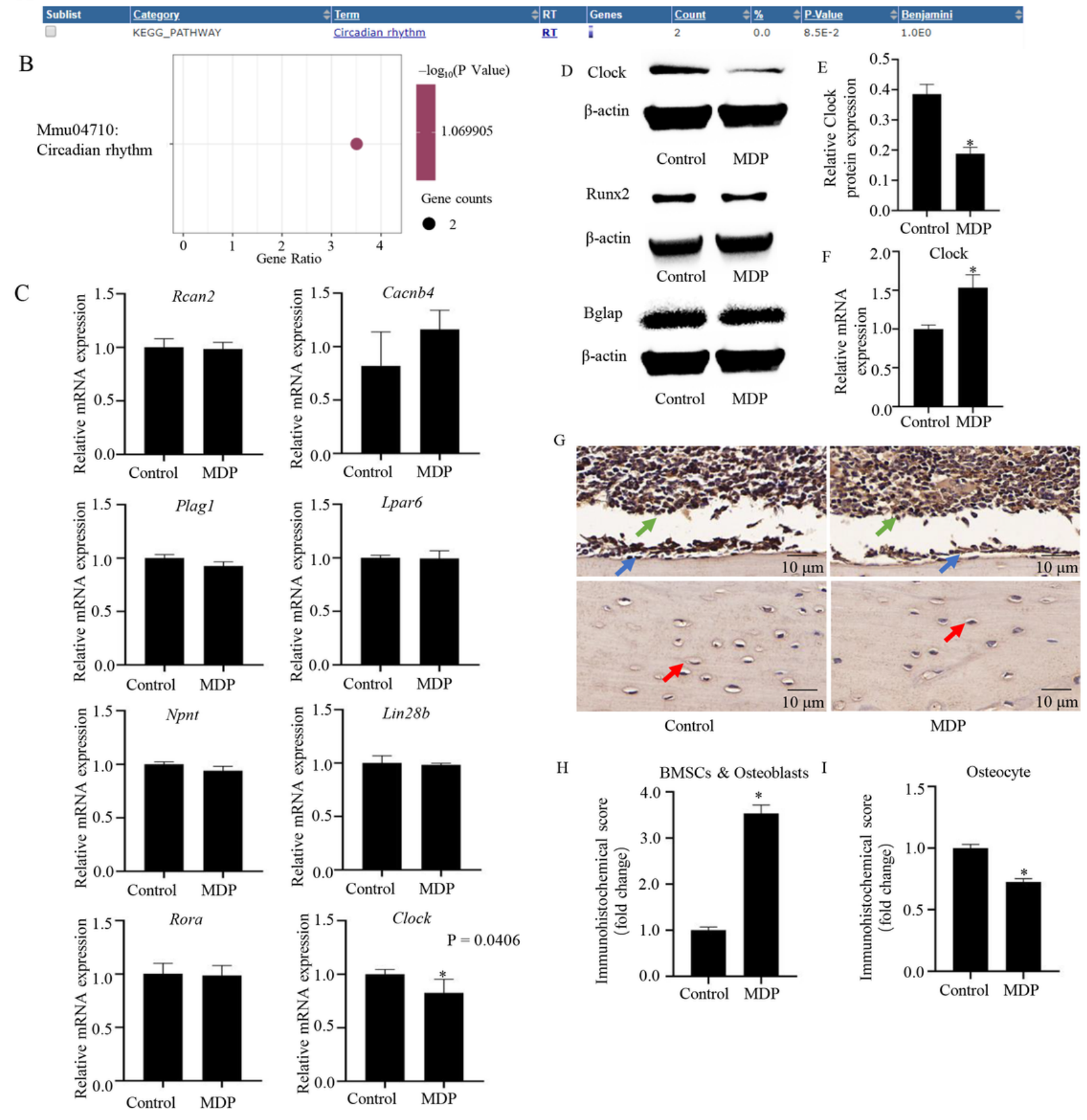

Figure 4

Bioinformatics analysis of the gene pathways that were enriched in the N.g MDP-treated osteocytes and verification in vitro. $(a, b)$ Pathway enrichment analysis showed that the differentially expressed genes, including clock and Rora, were associated with Circadian rhythm pathways. The size of the nodes and the intensity of the color indicate the gene number and mean P value. (c) qRT-PCR showed decreased expression of clock mRNA, n=3. (d) Western blots showed decreased expression of CLOCK, RUNX2, and Bglap. (e) Quantitative analysis of the expression of CLOCK protein. $n=3$. (f) qRT-PCR showed increased expression of clock mRNA after osteogenic induction. (g) In vivo, immunohistochemistry showed increased CLOCK expression in bone mesenchymal stem cells (BMSCs) and osteoblasts, but decreased expression in osteocytes. Scale bar $=10 \mu \mathrm{m}$. (h,i) Quantitative analysis of immunohistochemical score of (g). Green arrow, BMSCs; blue arrow, osteoblast; red arrow, osteocyte; Control, the 
sample was treated with culture medium in vitro or saline only in vivo; MDP, the sample was treated with $1 \mu \mathrm{g} / \mathrm{ml} \mathrm{N.g}$ MDP for $36 \mathrm{~h}$ in vitro or $2 \mu \mathrm{g} \mathrm{N.g} \mathrm{MDP} \mathrm{dissolved} \mathrm{in} 100 \mu \mathrm{l}$ of saline solution for 10 days in vivo.

\section{Supplementary Files}

This is a list of supplementary files associated with this preprint. Click to download.

- Supplementalfigure1.pdf

- Supplementalfigure2.pdf 